\title{
Minnesota Social Contacts and Mixing Patterns Survey with Implications for Modelling of Infectious Disease Transmission and Control
}

\author{
Audrey M. Dorélien ${ }^{1}$, Alisha Simon ${ }^{2}$, Sarah Hagge ${ }^{2}$, Kathleen Thiede Call', Eva Enns ${ }^{1}$, Shalini Kulasingam ${ }^{1}$ \\ ${ }^{1}$ University of Minnesota, ${ }^{2}$ Minnesota Department of Health \\ Keywords: mathematical models of infectious diseases, age-specific contact matrices, sars-cov-2, mixing patterns, social contacts \\ https://doi.org/10.29115/SP-2020-0007
}

Survey Practice

Vol. 13, Issue 1, 2020

\begin{abstract}
Emerging infectious diseases, such as the 2019 novel coronavirus (SARS-CoV-2), pose a substantial challenge to United States (US) public health. In the absence of a vaccine, controlling the spread of SARS-CoV-2 depends on social distancing measures, such as school closures and stay-at-home orders. Infectious disease epidemiologists create models to test the effects of these different interventions and to predict their impact on different populations. One key input to these models is data on social contact and mixing patterns. Unfortunately, there is a paucity of this type of data for the US. The Minnesota Department of Health and University of Minnesota launched the Minnesota Social Contact Study (MN SCS) in order to capture social contact and mixing pattern data for $\mathrm{MN}$ as different social distancing measures are enacted. This report describes the MN SCS survey and survey methodology. We highlight key differences between the MN SCS and the most widely cited and used social contact survey based on data from eight European countries in 2006. We conclude by highlighting changes others may consider when adopting the survey for use in other populations. A copy of the survey instrument is included in the appendix.
\end{abstract}

\section{Introduction}

The first positive case of the 2019 novel coronavirus disease (COVID-19) caused by the SARS-CoV-2 virus in Minnesota (MN) was confirmed on March 6,2020 . By June $3 \mathrm{rd}$, the number of confirmed cases had increased to 25,870 , with 1,086 lab confirmed deaths (Minnesota Department of Health n.d.). On March 13, 2020 MN Governor Tim Walz signed an Executive Order 20-01 declaring a peacetime emergency mandating that Minnesotans stay at home and practice social distancing. Starting May 18, 2020 the stay-at-home order was replaced by a less restrictive stay-safe order. A similar situation has been playing out throughout the United States (US). In the absence of a vaccine, MN and other states must rely on non-pharmaceutical interventions (NPIs) that seek to reduce contacts, such as school closures, physical distancing, and stay-at-home orders, to control the disease. 
The Minnesota COVID-19 Modeling effort is a collaboration between the University of Minnesota School of Public Health and the Minnesota Department of Health $(\mathrm{MDH})$. The model predicts the trajectory of the outbreak and allows us to test the effects and consequences of different COVID-19 intervention measures and to see how it may affect the number of cases, deaths, and the availability of intensive care unit (ICU) beds. A key input to the model is data summarizing "social contact" (contacts in close proximity, long enough to exchange three or more words, or that are physical in nature) patterns that are important for disease transmission; however, these data are not currently available for the US, let alone in MN. In order to parametrize the model using locally relevant data, the $\mathrm{MDH}$ and researchers at the University of Minnesota launched the Minnesota Social Contact Study (MN SCS).

In the next few sections, we highlight the importance of social contact and mixing pattern data for infectious disease models and the need to collect this information during different social distancing measures, and we review what data currently exists for the US. The second section describes the MN SCS and provides key details on the survey methodology. Section three concludes and provides some recommendations. We include a copy of the survey in the appendix.

\subsection{US COVID-19 models lack critical information on social contact and mixing patterns}

For COVID-19, understanding contact and mixing patterns is of critical importance because different NPIs aimed at reducing contacts have been instituted across the US. Modeling is being used to project how these different NPIs may affect the spread of SARS-CoV-2 through the US population. Given that each state and local government has had to determine what measures to institute and when, there is an urgent need for accurate, localized social contact pattern data.

Specifically, estimates of age-specific daily contacts are a critical input in mathematical models of SARS-CoV-2, because clinical outcomes and transmission can potentially vary by age. Unfortunately, we do not have data on usual daily contact patterns and how these vary by age and context for the US, let alone for each state or local area. Further, we do not know how contact patterns are changing under different mitigation strategies, the level of adherence or whether there are compensating contacts, such as playing with neighborhood children instead of children at school or grandparents looking after grandchildren at home.

\subsection{Use of social contact data in infectious disease models}

Due to the lack of age-specific contact data, US SARS-CoV-2 models make assumptions about age-specific transmission rates, such as assuming that anyone can transmit the infection to anyone else with the same probability or assuming that people spend much more time with other people of similar 
age and gender (Del Valle et al. 2007). This is problematic because studies have shown that models that are based on these assumptions without age contact data are not as accurate (Wallinga, Teunis, and Kretzschmar 2006). Alternatively, epidemiologists have used age-specific contact data from other countries which may not be representative of the US (Ferguson et al. 2020). Consequently, researchers have stressed the need for empirically-based estimates.

Fortunately, according to the "social contact hypothesis", age-specific transmission rates can be proxied by multiplying the age-contact matrices obtained from social contact surveys (see Figure 1) by a proportionality factor, which captures potential rates of infection and susceptibility (Wallinga, Teunis, and Kretzschmar 2006; Beutels et al. 2006; Edmunds, O'callaghan, and Nokes 1997; Mossong et al. 2008). The minimum amount of information needed to generate an age-contact matrix is information on the age of respondents and the age of each of their contacts during the course of a day. In the example in Figure 1, respondents (the person whose contact information is being reported; adults report for themselves or young children whose information is reported by a household adult) are organized in ten-year age groups and their contacts are also organized in ten-year age groups. The age-contact matrices $(\mathrm{M} i j)$ display the average number of daily contacts (e.g., close proximity conversation and/or physical contact) that respondents in one age group (i) have with individuals in another age group $(j)$. These matrices can capture many of the important components of social contact structures, such as typical household composition and daily routines and activities (e.g., school).

\subsection{The existing social contact data for the US is limited}

Two studies have used American Time Use Survey (ATUS) data to estimate some aspects of social contact/mixing patterns for the US. However, because both relied on time use surveys as opposed to social contact surveys, they are missing information on the number of contacts and the ages of respondents' contacts. The earlier of the two studies used data from the 2003 ATUS combined with the 1989-1990 Activity Pattern Survey of California Children and the 1987-88 Activity Pattern Survey of Californians, to create "duration of exposure matrices" for household and non-household locations. Since there is no information on the gender and age of non-household contacts, they had to use the proximate mixing assumption to estimate the ages of non-household contacts. They were not able to generate matrices with the mean number of contacts (Zagheni et al. 2008).

More recently, Dorélien et al. (2020) used ATUS data from 2003 to 2018 to describe the mean number and mean duration of social contacts taking place at home by respondents' age (Dorélien, Ramen, and Swanson 2020). They were also able to estimate the age-specific mixing matrices showing the mean number and mean duration of contacts between household members. For locations outside of the respondents' home, they were only able to describe the 


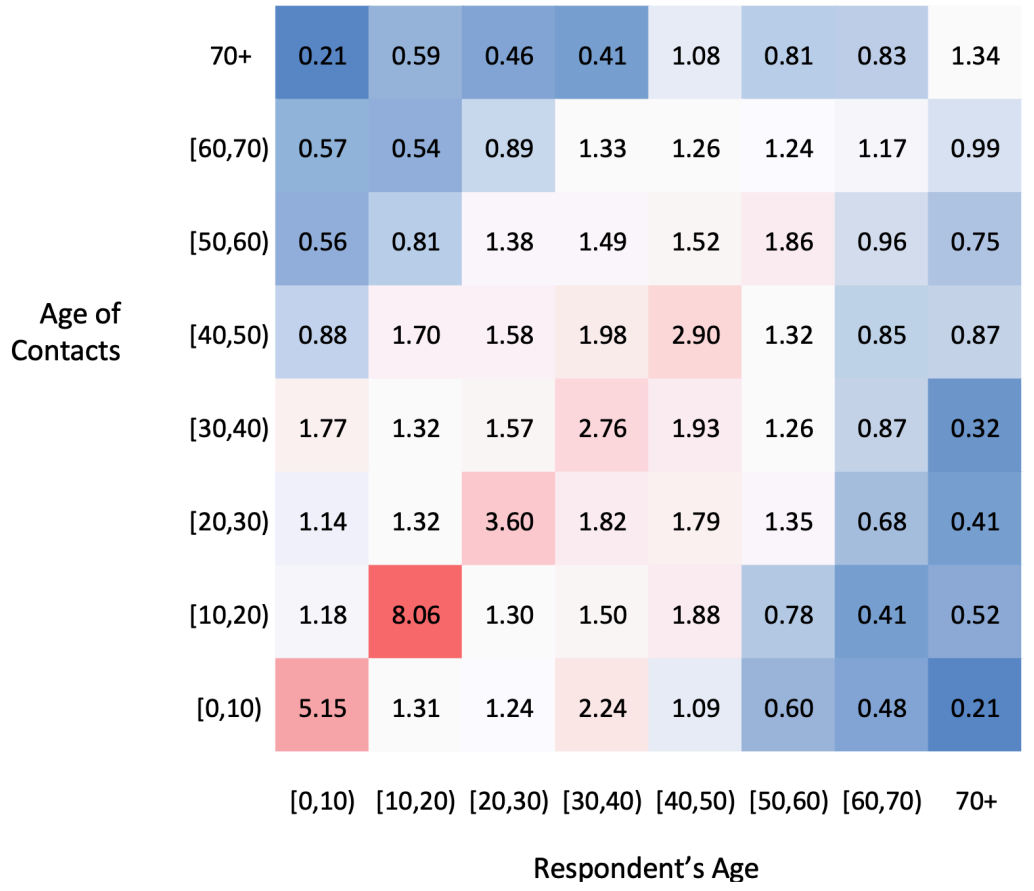

Figure 1: Age-contact matrix for the United Kingdom

Generated by the authors from the POLYMOD (Improving Public Health Policy in Europe through Modelling and Economic Evaluation of Interventions for the Control of Infectious Diseases) social contact surveys (Mossong et al. 2008). This matrix does not weigh for type of day (week/weekend).

age-pattern of the duration of social contacts. Since ATUS participants were all over the age of 15 , information is missing for younger age groups, which is pivotal for informing the effectiveness of school-based mitigation strategies or stay-at-home strategies.

\subsection{Minnesota Social Contact Study (MN SCS)}

The aim of the MN SCS is to update and adapt the most widely used and cited social contact survey, the POLYMOD (Improving Public Health Policy in Europe through Modelling and Economic Evaluation of Interventions for the Control of Infectious Diseases) survey conducted by Mossong et al. (2008), for use in MN. The POLYMOD survey took place in eight European countries (described in detail in Section 2.4). The MN SCS recruited a telephone and address-based sample of people who live in MN to determine current contact patterns under the social distancing mandates that were in place at the time of survey. We plan to collect two or three waves of data to monitor patterns of social contact as policies continue to be enforced or change.

\section{Description of Survey and Details of Survey Methodology}

\subsection{Sample frame}

Participants in the MN SCS were recruited from participants who stated their willingness to be contacted again for a follow-up survey in the 2019 Minnesota Health Access Survey (MNHA) sample (completed August through 
December of 2019). The MNHA is a biennial dual frame (telephone and address-based) survey providing data on health insurance coverage, reports of access, affordability, and use of health care services; demographics; health status; and household composition. The MN SCS can be linked to MNHA responses, allowing us to both shorten the MN SCS and to strengthen the social contact data collected.

The MNHA uses a stratified random sample, oversampling MN households outside of the Twin Cities Metro area and households more likely to include people of color or American Indians. This sample design allows us to generate estimates at the subpopulation level, and reflect variation in social contact patterns by geography and other demographics.

The final 2019 MNHA sample included completed surveys from 11,533 households. Approximately 48\% (5,578) of these households completed the survey in English and had an adult participant willing to be contacted for follow-up studies. Households willing to be re-contacted were comparable to the original sample on a range of demographic and employment characteristics, residence in urban and rural areas of the state, household size and health insurance coverage.

A random sample of 2,790 households was drawn for Wave 1 of data collection, leaving 2,788 households for Wave 2 of data collection (or randomly split into equal parts to field Wave 2 and Wave 3 of data collection). Wave 1 of data collection, which took place during the stay-at-home order, began on April 17, 2020 and ended on May 7, 2020. A total of 1,602 households completed surveys in Wave 1. Fifteen households were dropped during data cleaning, resulting in a final sample of 1,587 households.

\subsection{Recruitment method}

Households are contacted in the same way they were contacted for the 2019 MNHA. An adult (18 years or older) is invited to complete the survey. Participants in the random digit dial (RDD) telephone sample are invited to complete the MN SCS by telephone, or online if requested. A maximum of four call attempts are made to contact RDD households. MNHA participants in the address-based sample (ABS) are recruited by mail. An adult is instructed to use the secure access code provided in the letter to participate in the MN SCS online; they also have the option to call and complete by phone. Nonrespondents to the initial invitation letter receive a postcard and follow-up letter. Although the MNHA is conducted in English and Spanish, only 14 Spanish-speaking participants agreed to be re-contacted. With an assumed response rate of $40 \%$, translating the MN SCS is cost prohibitive, so it is available only in English. Telephone interviews are scheduled to occur at varied times of the day and varied days of the week to reflect differences in contact 
patterns throughout the week. To increase the odds that ABS households complete the social contact survey on varied days of the week, each sample wave is divided into three mailings posted every other day.

\subsection{Financial incentives}

Consistent with the MNHA, a $\$ 2.00$ pre-incentive will be included with initial invitation letters to the $\mathrm{ABS}$ sample. This pre-incentive is consistent with industry standard and leads to higher response rates. RDD cell phone participants are offered $\$ 5.00$ remuneration. This is important as some cell phone owners have "pay as you go" or limited minute contracts.

\subsection{Description and justification for key survey questions}

A team of survey researchers and infectious disease modelers revised and expanded the original POLYMOD survey to ensure relevant data were gathered (Mossong et al. 2008). Respondents are asked to report contacts made during the previous day (yesterday). Because we are interested in contacts that can result in infectious disease spread, a contact is defined as $\mathbf{1}$ ) either a two-way conversation with three or more words in the physical presence of another person, or for children who are not yet speaking, a one-way conversation in the physical presence of another person; or 2) physical skinto-skin contact (for example a handshake, hug, kiss or contact sports).

For every recorded contact, the respondents are asked to record the age (minimum information needed to create a basic contact matrix), gender, duration (probability of transmission increases with duration)(De Cao et al. 2014), the frequency with which they usually contact this person, whether contact was physical (skin-to-skin) or not (a measure of the intensity of contact), and the location where the contact occurred (home, school, work, transportation, etc.). Information on gender allows us to determine if differences in contact patterns can explain any differences in the risk of acquiring infection by gender. Information on location allows us to generate age contact matrices for specific locations. Contact matrices by location allow us to quantify the impact that different NPIs such as closure of schools and working from home have on transmission.

We are also interested in studying the determinants of differences in contact patterns; therefore, we collected data on household size and employment status, as well as a measure of current symptoms (e.g., loss of smell, taste, fever, etc.). Linking to the MNHA data also provides a host of sociodemographic data on respondents, including race, ethnicity, income, education, marital status, and industry of employment.

\subsection{Survey design}

The POLYMOD survey is a prospective self-administered paper survey in the form of a daily diary beginning at 5am on the day of the week the participant is assigned. The survey was altered to be a retrospective for the MN SCS 
because a portion of the MNHA sample requires an interviewer to collect these data by telephone. In both sampling frames, adult participants are asked to provide social contact information for the previous day. Households with children under age 18 are asked to provide social contact information for a randomly selected child (child with the most recent birthday), followed by a request for the adult to continue and provide contact data for themselves. Consequently, the survey sample will result in a larger number of respondents than households.

The survey begins by asking for a count of people currently living or staying in the household, the age, gender, and employment status of the adult participant, and the ages of other people in the household. Consistent with the POLYMOD survey, an introductory paragraph defines social contact and gives an example of information that will be requested, including how to count time.

The MN SCS allows for complete contact information (i.e., information on contact age, gender, nature, duration, frequency, and location of contact) on up to 30 contacts per person (child and adult in households with children; one adult in adult-only households), and for abbreviated contact information for large numbers of contacts in childcare/school or work settings. Concerns about participant burden in providing contact information for as many as 30 people over the telephone led to the decision to begin by asking about social contacts in potentially larger group settings first (childcare/school and work) followed by other settings. For simplicity, we describe this for adult-only households. For college and work settings separately, participants are asked how many people they had contact with. If participants had contact with ten or more people, they are asked how many of the contacts from that setting they a) interacted with on a daily or almost daily basis and b) how many of the contacts they had physical contact with such as a handshake, hug, or contact sports. If participants had contact with fewer than ten contacts at college or work, they are asked to enumerate and describe each contact including the contact's approximate age, gender, location (at home or in the yard, childcare/ school/college, work, transport, outdoors away from home, grocery or other store, etc.); duration (increments from under 5 minutes, 5-15 minutes, more than 15 minutes to 1 hour, more than 1 hour to 4 hours, to more than 4 hours); frequency (e.g., daily or almost daily, about once or twice a week, about once or twice a month, less than once a month, or this was the first time they met); and whether physical skin-to-skin contact occurred. Additionally, participants are asked about the number of contacts they had outside of school or work settings and are asked to enumerate and describe each of these contacts.

The survey ends by asking whether the participant had any of a series of symptoms on the previous day (i.e., fever, chills, cough, shortness of breath, sore throat, headache, muscle aches, diarrhea, lost sense of taste, or lost sense 
of smell), checking all that apply. This serves the dual purpose of allowing us to determine if contacts are reduced when someone displays symptoms and syndromic surveillance for the COVID-19 virus.

\section{Discussion and Conclusion}

In conclusion, we describe how we adapted the POLYMOD surveys developed to measure contact patterns in Europe to be administered online and by telephone for a US context. These data are critical for informing infectious disease models of the spread of SARS-CoV-2 in different US populations and the impact that currently recommended NPIs will have on reducing spread.

We recommend that for individuals with 10 or more work or school contacts, that information is also collected on how many of those contacts fall within 10 -year age groups starting from 0 to 80 or older. This language is missing in the first wave of data but we strongly recommend adding aggregate age questions to similar social contact surveys in order to create complete agecontact matrices.

There are limitations, however. Understanding the contact and mixing patterns of children is essential for characterizing the transmission of many infectious diseases. In the MN SCS, an adult/parental proxy responds on behalf of children under the age of 18 . This may lead to a bias in the estimation of contacts for younger age groups. In the majority of the POLYMOD surveys, parental proxy reporting was used for children ages 0-8; children ages 9-17 were identified as self-reporting (Mossong et al. 2008). To address this, we included a section in the instructions that encourages the adult to ask children for input.

The MN SCS did not include COVID-19 specific questions. Nevertheless, the last question on the survey asks if participants had any symptoms the day of the survey that are potentially associated with COVID-19. Respondents may have different mixing and contact patterns when feeling ill versus healthy; consequently, asking about health status may allow us to be better able to identify contacts that actually lead to transmission. Furthermore, this type of information has been asked in other social contact surveys (Hoang et al. 2019; Stein et al. 2014; DeStefano et al. 2011; Willem et al. 2012). Other factors may also influence respondents' contact patterns, such as the presence of high-risk individuals in the household; however, these factors do not necessarily increase the probability that a contact leads to disease transmission. Our intention is to create a survey instrument that can be repeatedly administered, not just during the COVID-19 pandemic. Other recent social contact surveys have asked questions such as attitudes towards COVID-19 and the effect of physical distancing measures and whether participants or household members had COVID-19 symptoms, were tested for COVID-19, or had contacts with known cases (Jarvis et al. 2020). 
As part of an existing study, the MN SCS already had information on respondents' race and ethnicity, as well as income and homeownership. We recommend future versions of the survey include demographic questions so that we can better understand how these differences affect contact patterns as well as ability to stay home or adopt different NPIs.

Finally, current mitigation strategies have distinguished between essential workers (defined as workers in critical sectors, such as healthcare and public health, law enforcement, public safety, first responders and food/agriculture, who are preforming work that cannot be done in their residence through telework) and non-essential workers. We recommend that future iterations of the survey include occupational codes to allow researchers to distinguish between essential and non-essential workers. Information on occupational codes could help us identify which occupations are associated with a large number of contacts in the workplace and how these workers mix with others outside of the workplace, which could help improve mitigation measures to control disease spread.

\section{Acknowledgements}

Dorélien was supported by the Minnesota Population Center P2C HD041023 grant and a grant from the University of Minnesota, Office of the Vice Provost for Research. Drs. Call, Enns and Kulasingam are supported by a grant from the Minnesota Department of Health and the University of Minnesota, Office of the Vice Provost for Research.

\section{Corresponding author info:}

Audrey Dorélien 30119 th Ave S, Minneapolis, MN 55455

Email: dorelien@umn.edu

Tel: 612-626-7403 


\section{REFERENCES}

Beutels, P., Z. Shkedy, M. Aerts, and P. Van Damme. 2006. “Social Mixing Patterns for Transmission Models of Close Contact Infections: Exploring Self-Evaluation and Diary-Based Data Collection through a Web-Based Interface.” Epidemiology and Infection 134 (6): 1158-66. https://doi.org/10.1017/s0950268806006418.

De Cao, Elisabetta, Emilio Zagheni, Piero Manfredi, and Alessia Melegaro. 2014. "The Relative Importance of Frequency of Contacts and Duration of Exposure for the Spread of Directly Transmitted Infections." Biostatistics 15 (3): 470-83. https://doi.org/10.1093/biostatistics/kxu008.

Del Valle, S.Y., J.M. Hyman, H.W. Hethcote, and S.G. Eubank. 2007. "Mixing Patterns between Age Groups in Social Networks." Social Networks 29 (4): 539-54. https://doi.org/10.1016/ j.socnet.2007.04.005.

DeStefano, F., M. Haber, D. Currivan, T. Farris, B. Burrus, B. Stone-Wiggins, A. McCALLA, et al. 2011. "Factors Associated with Social Contacts in Four Communities during the 2007-2008 Influenza Season.” Epidemiology and Infection 139 (8): 1181-90. https://doi.org/10.1017/ $\underline{s 095026881000230 \mathrm{x}}$.

Dorélien, Audrey M., Aparna Ramen, and Isabella Swanson. 2020. "Analyzing the Demographic, Spatial, and Temporal Factors Influencing Social Contact Patterns InUS and Implications for Infectious Disease Spread.” http://www.audreydorelien.com/atus_social_contact_latest.

Edmunds, W. J., C. J. O'callaghan, and D. J. Nokes. 1997. "Who Mixes with Whom? A Method to Determine the Contact Patterns of Adults That May Lead to the Spread of Airborne Infections." Proceedings of the Royal Society of London. Series B: Biological Sciences 264 (1384): 949-57. https://doi.org/10.1098/rspb.1997.0131.

Ferguson, N, D Laydon, G Nedjati Gilani, N Imai, K Ainslie, M Baguelin, S Bhatia, et al. 2020. "Report 9: Impact of Non-Pharmaceutical Interventions (NPIs) to Reduce COVID19 Mortality and Healthcare Demand.” Imperial College London. https://doi.org/10.25561/77482.

Hoang, Thang, Pietro Coletti, Alessia Melegaro, Jacco Wallinga, Carlos G. Grijalva, John W. Edmunds, Philippe Beutels, and Niel Hens. 2019. "A Systematic Review of Social Contact Surveys to Inform Transmission Models of Close-Contact Infections.” Epidemiology 30 (5): 723-36. https://doi.org/10.1097/ede.0000000000001047.

Jarvis, Christopher I., Kevin Van Zandvoort, Amy Gimma, Kiesha Prem, Megan Auzenbergs, Kathleen O’Reilly, Graham Medley, et al. 2020. "Quantifying the Impact of Physical Distance Measures on the Transmission of COVID-19 in the UK." BMC Medicine 18 (1): 124. https://doi.org/10.1186/s12916-020-01597-8.

Minnesota Department of Health. n.d. "Situation Update for COVID-19." Accessed June 5, 2020. https://www.health.state.mn.us/diseases/coronavirus/situation.html.

Mossong, Joël, Niel Hens, Mark Jit, Philippe Beutels, Kari Auranen, Rafael Mikolajczyk, Marco Massari, et al. 2008. "Social Contacts and Mixing Patterns Relevant to the Spread of Infectious Diseases.” Edited by Steven Riley. PLOS Medicine 5 (3): e74. https://doi.org/10.1371/ journal.pmed.0050074.

Stein, Mart L., Jim E. van Steenbergen, Vincent Buskens, Peter G. M. van der Heijden, Charnchudhi Chanyasanha, Mathuros Tipayamongkholgul, Anna E. Thorson, Linus Bengtsson, Xin Lu, and Mirjam E. E. Kretzschmar. 2014. "Comparison of Contact Patterns Relevant for Transmission of Respiratory Pathogens in Thailand and the Netherlands Using RespondentDriven Sampling.” Edited by James M. McCaw. PLOS ONE 9 (11): e113711. https://doi.org/ $\underline{10.1371 / \text { journal.pone.0113711. }}$. 
Wallinga, Jacco, Peter Teunis, and Mirjam Kretzschmar. 2006. "Using Data on Social Contacts to Estimate Age-Specific Transmission Parameters for Respiratory-Spread Infectious Agents." American Journal of Epidemiology 164 (10): 936-44. https://doi.org/10.1093/aje/kwj317.

Willem, Lander, Kim Van Kerckhove, Dennis L. Chao, Niel Hens, and Philippe Beutels. 2012. "A Nice Day for an Infection? Weather Conditions and Social Contact Patterns Relevant to Influenza Transmission.” Edited by Cécile Viboud. PLOS ONE 7 (11): e48695. https://doi.org/10.1371/ journal.pone.0048695.

Zagheni, Emilio, Francesco C. Billari, Piero Manfredi, Alessia Melegaro, Joel Mossong, and W. John Edmunds. 2008. "Using Time-Use Data to Parameterize Models for the Spread of Close-Contact Infectious Diseases.” American Journal of Epidemiology 168 (9): 1082-90. https://doi.org/ $\underline{10.1093 / \mathrm{aje} / \mathrm{kwn} 220 .}$. 


\section{SUPPLEMENTARY MATERIALS}

\section{Minnesota Social Contact Study (WAVE 1)}

Download: https://www.surveypractice.org/article/13669-minnesota-social-contacts-and-mixingpatterns-survey-with-implications-for-modelling-of-infectious-disease-transmission-and-control/ attachment/38748.docx 